\title{
A Novel Predictive Current Control For Active Power Filter
}

\section{Dake Gao, JingRong Yu}

Central South University, Power Electronics And Renewable Research Institute, ChangSha, China 515037030@qq.com

Keywords: active power filter; delay; predictive current control ; nonlinear inductor

Abstract. The zero-order hold, data-sampling and data-processing of digital controller may cause inevitable control delay in active power filter, which not only affects the harmonic compensation performance, but also causes the instability of active power filter. In order to eliminate the delay effect, a novel predictive control combined with reference current prediction based on proportion resonance and system model prediction is proposed. The value of inductor changes nonlinearly when the active power filter outputs harmonic current of different orders, while the parameters of prediction control are designed according to the inductor parameters. To eliminate the influence of nonlinear inductor, the function of the inductor parameter changes by the inductor current is constructed, and the control parameters of predictive control are modified according to the function.This method effectively suppresses model errors caused by nonlinear inductor , and increases the speed and accuracy of current prediction. Simulation and analysis of several current control methods verify the validity and feasibility of this current method.

\section{Introduction}

With the rapid development of power electronic technology, a large number of variable flow devices are widely used in power system,which leads to the increasingly serious problem of harmonic pollution in power system. As a device for dynamic harmonic suppression and reactive power compensation, active power filter (APF)can produce compensating current offset load,current harmonics and reactive components in real time, which ensures the safe and reliable operation of the power grid and equipment.The existing APFs use digital controller to realize the zero steady state error tracking of each harmonic wave. However, the delay effect generated during the digital control 
process makes the stability margin of the active filter very narrow, and the anti-disturbance ability of the system is not strong.

As to the disadvantage of the delay of digital controller to the performance of the system, predictive control of time delay compensation is an effective way [1-4].Predictive control consists of two parts: reference current prediction and system model predictive control. Reference current prediction has different predictive control algorithms to improve the performance of the system with high reliability, and good robustness.

Literature [5] proposes to combine the adaptive prediction algorithm and interpolation algorithm to realize the reference current prediction, to reduce the steady-state prediction diviation, and to impove prediction precise.Predictive control can improve the stability and improve the stability and dynamic performance of the system.

With regard to grid connected inverter, literature [6-7] proposes a new deadbeat current predictive control, adding compensation control to improve the control performance, to faster the response speed and to reduce the current distortion rate . In literature [8], the model predictive control is adopted for the energy storage inverter. The added predictive error suppression control effectively elimiates the effect of the error on the control performance. However, the model predictive control depends on the accuracy parameters of the system. Too much model deviation can not improve the performance, what' s more, the system will be instable.So the accuracy of the model is very necessary, and nonlinear factor of the inductance has a great influence on the accuracy of model.

As for the permanent magnet synchronous motor, a robust current predictive control algorithm is proposed inliterature [9]. By establishing the state equation and discretization of the predictive reference current, the Luenberger state observer is used to predict the actual output current, and it is proved that the large inductor error can cause the system divergence.

In this paper, the predictive current control based on the system model is proposed, which can both predict the reference current and the compensation current. In the predictive control, if the inductor is linearized, the accuracy of the model will be not enough and the prediction error of the compensation current will be too large. Because when the inductor current get larger, the inductor value will be 
obviously decreased. In this paper, the nonlinearity of the inductor is compensated by the predictive control , the large model error is avoided, and the compensation current is predicted quickly and accurately. The harmonic compensation performance of the system is the best.

\section{Model of Active Power Filter}

The model of shunt active power filter is shown in Figure 1. The main circuit of APF is composed of LCL filter and inverter circuit. In the figure, $U_{g}$ is the phase voltage of the grid, $I_{g}$ is the phase current of the grid , $i_{L}$ is the phase current, load current is nonlinear, and $U i$ is the phase voltage of the inverter side. APF generates compensation current $I$ that is equal to the magnitude of the load harmonic current, which ensures that the current $I_{g}$ of the network is more to the sine wave. The control of the compensation current and the real-time tracking are the key of the APF.

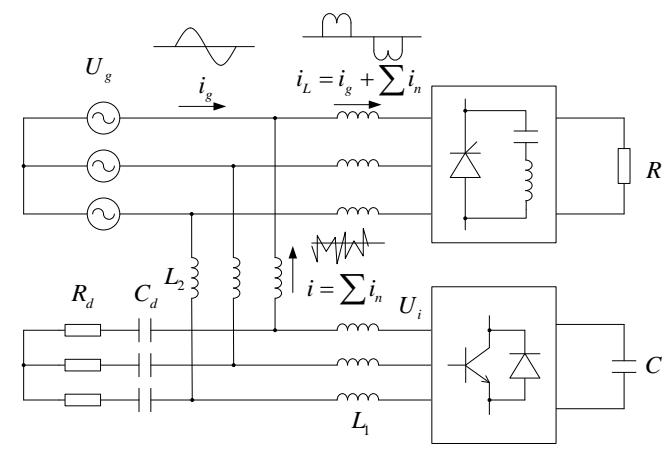

Fig.1 System model of the three-phase shunt APF

\section{Traditional current control analysis}

Time Delay Analysis.The digital controller is calculated to obtain the reference voltage output value of the modulated wave from a certain time according to sampling current, and the output value of the digital controller is kept constant by the zero order holder until the output of the next sampling. The sampling, calculation and output conversion of an ideal controller are considered at the same time point. But in fact, the data calculation and processing inevitably lead to the system delay deviation, which will not only affect the accuracy of the compensation current, but also may cause the system to be unstable. The delay of the digital controller is as shown in Figure 2, the sampling apparatus samples the load current value and the output referenced current value after calculation. $k T s$ moment 
is the PWM loading point, Ts is both the sampling period and the controller's control period.tc is the sampled data computation time.and $t d$ is the system delay time. It can be seen from the figure that the compensation current does not track the load harmonic current in real time, and the time lag causes the system to become worse. The length of the delay is related to the speed of the controller.The minimum is $t c$, and the maximum is the sum of Ts and tc [13]. Usually, $\mathrm{n}$ sampling periods are reserved in the DSP for data processing. In other words, the time delay is n control cycle.

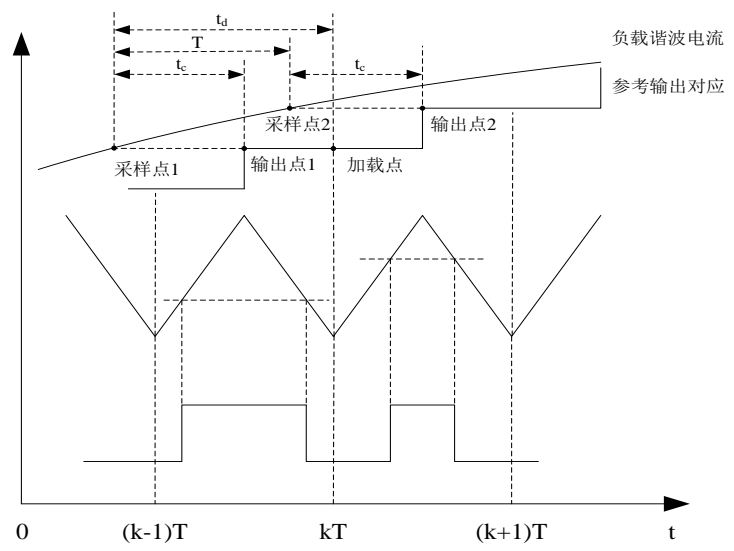

Fig.2 Delay diagram of the digital controller

Also for the controller's control period, $t c$ is the sampled data computation time, and $t d$ is the system delay time. It can be seen from the figure that the compensation current does not track the load harmonic current in real time, and the time lag causes the system to become worse. The length of the delay is related to the speed of the controller.The minimum is tc, and the maximum is the sum of Ts and $t c[10]$. Usually, $\mathrm{n}$ sampling periods are reserved in the DSP for data processing. In other words, the time delay is n control cycle.

Traditional Current Control Method. APF traditional current control is shown in Figure 3.As the PI controller can not achieve the zero steady-state deviation by responding to the sinusoidal signal, the current controller uses a proportional resonant control. Among them, Gpwm(s) is thetransfer function of voltage source PWM inverter, Gvi(s) is the transfer function of inverter LCL filter, Gd(s) is the transfer function of digital controller delay, and $G P R(s)$ is a transfer function of proportional resonant controller . Their transfer functions can be expressed as: 


$$
\left\{\begin{array}{l}
G_{d}(s)=e^{-n s T} \\
G_{p w m}=\frac{K_{p w m}}{\mu T_{s} s+1} \\
G_{v i}(s)=\frac{1}{s L+R} \\
G_{P R}(s)=k_{p}+\sum \frac{2 k_{n} s}{s^{2}+\omega_{n}^{2}}
\end{array}\right.
$$

\section{New Predictive Current Control Strategy}

Inductor Nonlinearity. Ferromagnetic material is selected for the inductor of APF main circuit for better performance. Due to the saturation effect of the core, the inductor value is not constant. The experimental curves of the 150uH Ferromagnetic inductors are shown in Fig 4. The average inductor values is L1 and the effective value I of the inductor current $\mathrm{i}$ are non-linear.Their relationship can be expressed as

$a L^{2} I^{2}+b L I+c=0$

The APF control circuit samples the load current and separates the harmonic current as the reference current of the inductor. According to the relationship between the valid value of reference current and the inductor value, the sliding window average algorithm is used to get the inductor value. Sliding window average algorithm is to take a reference current valid value in each same time interval, and the average of the finite set of valid values is the current value of the inductance.By removing the first effective value and adding the newly acquired effective value, the average value of the new set of valid values will be the inductor values of next moment.

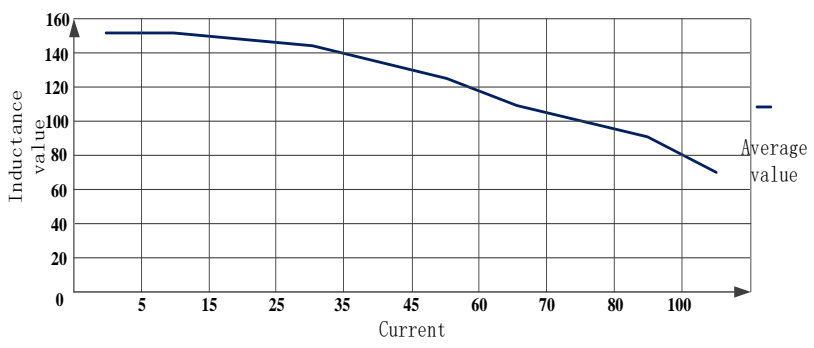

Fig. 3 Inductor $L$ versus $I$ 
It can be seen from Figure 3 that the inductorvalue of the reactor represents the nonlinear character according to effective value of the current.In the model predictive control, if the nonlinearity of the inductoris not considered, the inductor value will have a large deviation, the establishment of system model will be inaccurate, and the prediction current will have a great devistion. The consequences will be that compensation current can not accurately track the load harmonic current, the system's harmonic compensation performance will get worse, and too much inductor deviation may also lead to system instability. So it is very necessary to get the appropriate nonlinear fitting of the inductor.The nonlinear fitting of $\mathrm{L}$ can be expressed as $0.017+5.16 \times 10^{7} L I-3.67 \times 10^{16} L^{2} I^{2}=0$

According to the inductor value in the simulation, we can get the value of the corresponding $L$ is $170 u \mathrm{H}$.

New predictive current control. The digital controller has a time delay of a control period, which affects the stability and control performance of the system. In this paper, the control strategy is proposed based on the combination of reference current prediction and model prediction. Recursive Fourier analysis is used in the reference current prediction, or harmonic synchronous rotating reference frame.Each harmonic is detected, then multiply by $e^{j \omega T}$, then we get $i_{\text {habc }}^{\prime}$, it can be expressed as Eq.4.

$$
\left\{\begin{array}{c}
C_{22} C_{32} i_{h n a b c}=i_{d n}+j i_{q n} \\
\left(i_{d n}+j i_{q n}\right) \cdot e^{j \omega t}=i_{d n}^{\prime}+j i_{q n}^{\prime} \\
C_{23} C_{22}^{\prime}\left(i_{d n}^{\prime}+j i_{q n}^{\prime}\right)=i_{h n a b c}^{\prime} \\
i_{h 1 a b c}^{\prime}+i_{h 2 a b c}^{\prime}+\ldots+i_{h n a b c}^{\prime}=i_{h n a b c}^{\prime}
\end{array}\right.
$$

So each harmonic in the Inverse Fourier transform is conveniently predicted in advance of a control period. The model predicts get the predicted feedback current $i(k+1)$ at the time of $(K+1) T$ by using the actual compensation feedback current $i(k)$ at time $k T$ and the system parameters. In this way, the reference signal and the actual feedback signal simultaneously advance a control cycle, which can achieve the same effect as the situation without delay, and optimize the performance of the controller .The inductor value is not a mutation. 
During a control cycle, the $L$ can be seen as a constant. Neglecting the capacitor, the relationship between LCL filter single-phase model voltage and current are as follows:

$L \frac{d i(t)}{d t}=U_{i}-U_{g}-R i(t)$

We can get :

$i(t)=e^{\frac{-R}{L}\left(t-t_{0}\right)} i\left(t_{0}\right)+\frac{1}{L} \int_{t_{0}}^{t} e^{\frac{-R}{L}(t-\tau)}\left(U_{i}-U_{g}\right) d \tau$

As the sampling frequency is much greater than the grid frequency, the $R T_{s}$ is much less than 1 . In a sampling period, it can be considered that $U_{i}(t)$ is a slow linear change, $U_{g}(t)$ remains unchanged. Order $t_{0}=k T_{s}, t=(k+1) T s, i(k)=i(k T s)$.

$U_{i p}(k)$ is average voltage of $U_{i}(t)$ during a sampling period , the discrete model is approximated by: $i(k+1)=i(k)+\frac{T_{s}}{L}\left(U_{i p}(k)-U_{g}(k)\right)$

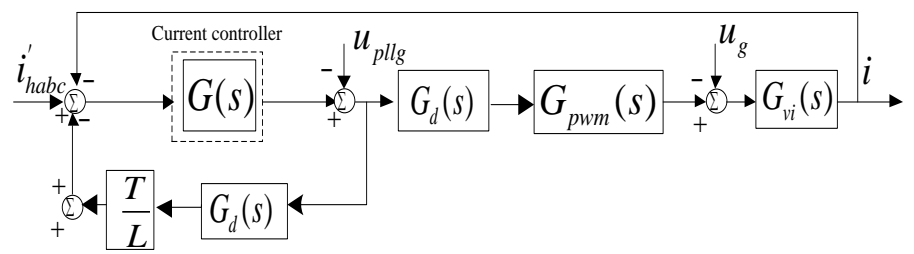

Fig. 4 predictive current losed-loop control diagram

So , the reference current $i_{\text {ref }}$ is calculated as the most faster tools to reach the hamonics .

From the above analysis, the transfer function block diagram of current prediction control is shown in Figure 4, the grid voltageis a disturbance to the current control , and the effect of voltage feed-forward is to offset the disturbance of grid voltage

\section{Simulation and analysis}

Simulation. Based on the simulation model of Matlab/Simulink software, the current and harmonic wave shape of the two control strategies are analyzed and compared . The system parameters are shown in Table 2, and the simulation waveforms are shown in Figure 5.It can be seen from the chart comparison, predictive current control have smaller grid current harmonics and stronger harmonic 
compensation ability compared with traditional current control, which is in accordance with the theoretical analysis.

Tab.2 System parameters of APF

\begin{tabular}{llll}
\hline Parameters & Figure & Parameters & Figure \\
\hline Grid line voltage/V & 380 & $R_{L} / \Omega$ & 8 \\
Grid frequency/Hz & 50 & $L_{1} / \mu \mathrm{H}$ & 20 \\
DC side voltage/V & 700 & $L_{2} / \mu \mathrm{H}$ & 150 \\
$R_{d} / \Omega$ & 3 & $C / \mu F$ & 7500 \\
$C_{d} / \mu F$ & 18 & $L_{3} / \mu \mathrm{H}$ & 40 \\
\hline
\end{tabular}

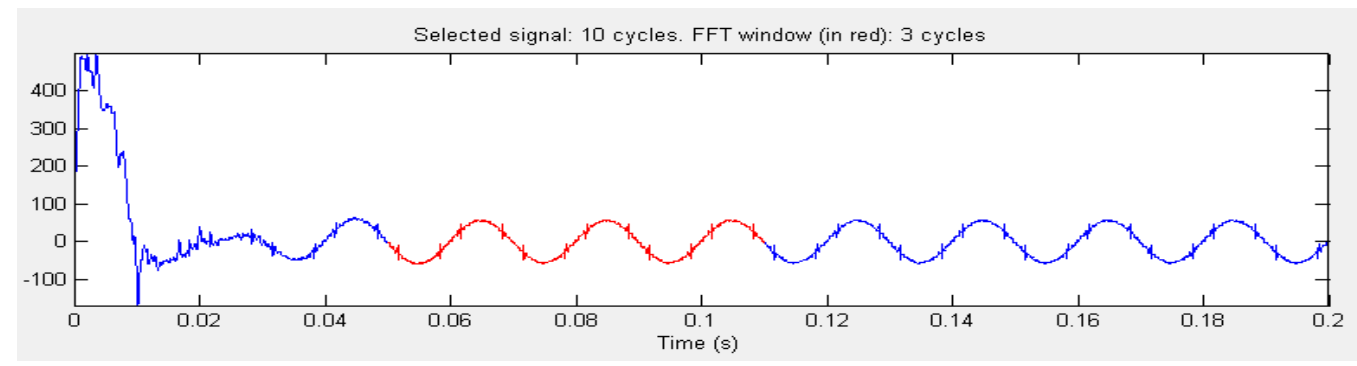

(a) Traditional current controlled grid current

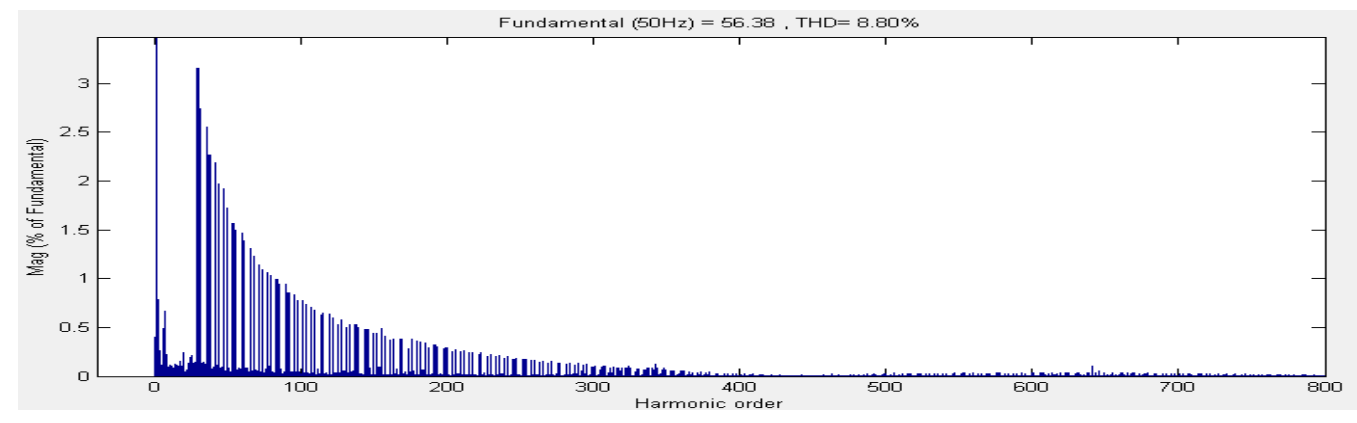

(b) Traditional current controlled grid current harmonics

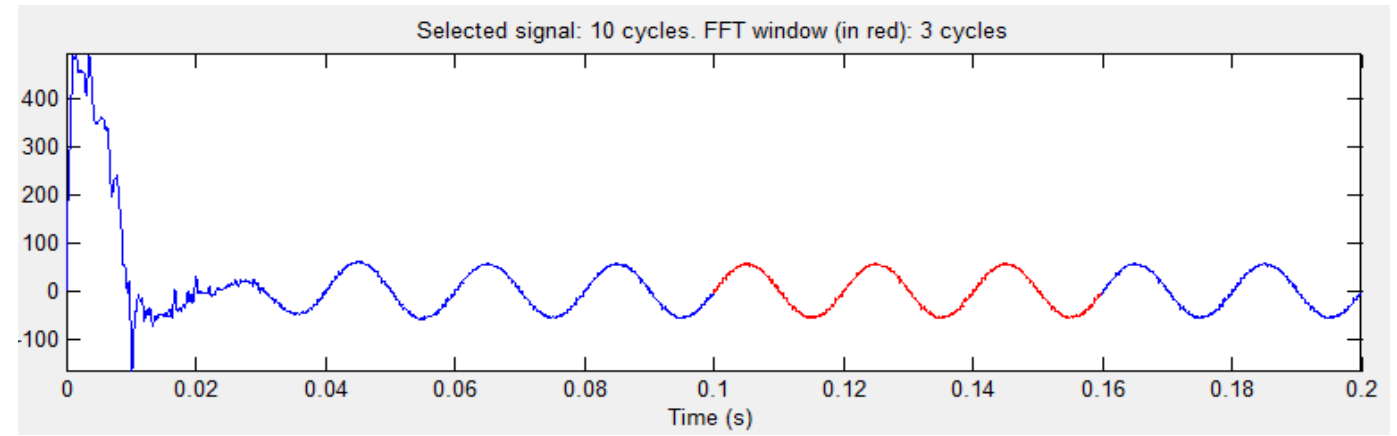

(c) Predicted current controlled grid current 


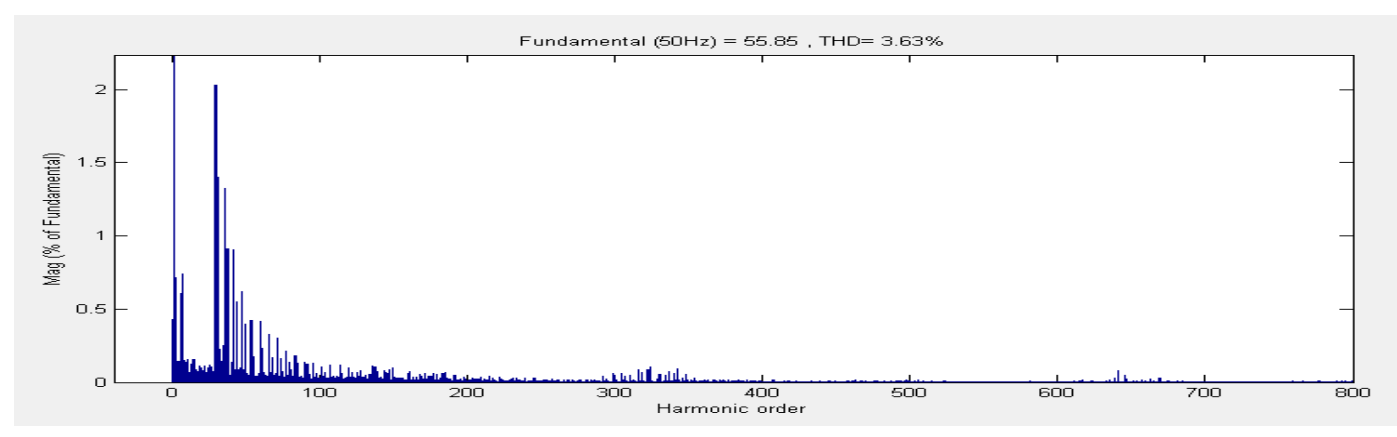

(d) Predicted current controlled grid currentharmonics

Fig. 5 Steady-state currents and Harmonics of i for two current Strategies

\section{Conclusions}

A predictive current control method based on proportional resonance is proposed in this paper to deal with the influenced hamonic compensation performance caused by control delay during the period of APF data processing.After simulation and experiment analysis, the conclusions can be drawn as follows:

In the case of time delay, the proportion coefficient of the system is relatively small, the cut-off frequency is low, and orders of the harmonic which needs compensation compensation is high, resulting in that the phase margin is very narrow and the system is easy to be instable.At the same time, the low cut-off frequency and not-too-high frequency of harmonic compensation greatly reduce the system's compensation performance. So it is very necessary to eliminate the delay.

Combining the reference current prediction and the model prediction, the delay effect of the load harmonic current and compensation current can be eliminated, which not only improves the performance of the system but also ensures the stability of the system. The current tracking ability is strong, steady and dynamic state effect is well, and reliability is high. Simulations verified the proposed effectiveness and superiority of the predictive current control .

In this paper, the sliding window average algorithm are used to fit the inductor value to prevent the overlarge current and the overlarge deviation in the compensation current forecast .Nonlinear processing of the inductor can reduce the error of model predict and maintain the stability of the system.The precision of current prediction is high, and the compensation performance is good.The 
feasibility and effectiveness of the proposed method are verified by comparison in the simulation and experiment diagram.

\section{References}

[1] Daniel Nahum Zmood,Donald Grahame Holmes. Stationary Frame Current Regulation of PWM Inverters With Zero Steady-State Error[J] . IEEE Transactions on power electronics,2003,18(3): 814-822

[2] Zhengyu Lyu, Zhaoming Qian.Neural network predictive control of shunt active power filter[J].Proceedings of The Chinese Society for Electrical Engineering,1999,19(12) : 6-10(In Chinese)

[3] Patricio Cortes,Jose Rodriguez,et al. Delay Compensation in Model Predictive Current Control of a Three-Phase Inverter[J]. IEEE Transactions on Industrial Electronics,2012,Feb,92(2) : $1323-1325$

[4] Jiang Li, Haishun Sun, Shijie Cheng, et al. Predictive control of active power filter based on Grey System Theory $[\mathrm{J}]$. Proceedings of The Chinese Society for Electrical Engineering,2002,22(2): 40-46(In Chinese)

[5] Mingyun Qi, An Luo, Dingguo Liu, et al.Prediction method and Realization of active power filter reference current[J] . Proceedings of The Chinese Society for Electrical Engineering,2009,29(7): 128-134(In Chinese)

[6] Yong Yang, Yi Ruan, Bingying Ye, et al.Deadbeat current predictive control method for three-phase grid connected inverter[J] . Journal of Chinese Electrical Engineering Science,2009,29(33): 40-46(In Chinese)

[7] Jaime Castelló Moreno, José M, et al. A Robust Predictive Current Control for Three-Phase Grid-Connected Inverters[J]. IEEE Transactions on Industrial Electronics， 2009，June，56(6): 1993-2003 
[8] Zhijian Fang, Shanxu Duan, Tianjin Chen, et al.Predictive control deviation formation mechanism and suppression strategy of energy storage inverter[J]. Proceedings of The Chinese Society for Electrical Engineering , 2013 , 33(30): 1-9(In Chinese)

[9] Li Niu, Ming Yang, Geng Wang, et al.Robust current control algorithm for permanent magnet synchronous motor based on Deadbeat Control[J]. Proceedings of The Chinese Society for Electrical Engineering,2013 , 33(15): 78-85(In Chinese)

[10] Xiangyang Xia, An Luo, et al.Novel compensation method for active filter control delay[J]. Transactions of China Electrotechnical Society , 2008 , 11(23) : 166-172(In Chinese) 